\title{
Isolation of Mycobacterium tuberculosis from different risk groups in and around Kakinada
}

\author{
K. Pragathi ${ }^{1}$, R. Lakshmi Kumari ${ }^{2}$, Durga Rani $^{3}$, Srinivas $^{4}$ \\ ${ }^{1}$ Dr. K. Pragathi, Lecturer, Department of Microbiology, Nizams Institute of Medical Sciences, Hyderabad, \\ ${ }^{2}$ Dr. R. Lakshmi Kumari, Professor and HOD, Department of Microbiology, Sidhartha Medical College, Vijayawada, \\ ${ }^{3}$ Dr. Durga Rani, Assistant Professor, Department of Microbiology, Rangaraya Medical College, Kakinada, ${ }^{4}$ Srinivas, \\ Lab Technician, Department of Microbiology, Rangaraya Medical College, Kakinada, Andhra Pradesh, India.
}

Address for Correspondence: Dr. K. Pragathi, Lecturer, Department of Microbiology, Nizams Institute of Medical Sciences, Hyderabad. E- mail: pkottapalli@hotmail.com.

\begin{abstract}
Introduction: Tuberculosis (TB) represents a global epidemic and causes significant mortality mostly in developing countries. With the emergence of HIV pandemic the steady decline in the incidence of tuberculosis was halted in developed countries and in fact it is reversed. Similarly an epidemic of diabetes mellitus is sweeping the country. Aims and Objectives: Isolation of Mycobacterium tuberculosis on solid media from different groups like HIV/AIDS patients, Diabetics, immunocompetent persons and to study the isolation rates in smear negative suspected cases. Materials and Methods: This study was performed on sputum of tuberculosis suspected patients from different risk groups. All the specimens were examined microscopically by using Ziehl-Neelsen's staining and decontamination was performed using Modified Petroffs technique. The decontaminated deposit was cultured on LJ slopes. Results: Out of a total of 50 HIV positive cases, 25 diabetic cases and 25 immunocompetent persons with suspected tuberculosis $44 \%, 8 \%$ and $68 \%$ were found to be co-infected with tuberculosis. Out of total of 100 cases of symptomatic pulmonary tuberculosis, 34 (34\%) were sputum positive and $66(66 \%)$ were sputum negative. Maximum no. of sputum positive cases are among symptomatic immunocompetent persons (60\%) followed by HIV positive cases (34\%) and Diabetics (8\%). All the strains isolated were identified as Mycobacterium tuberculosis and no non-tuberculous mycobacteria spp (NTM) were isolated. Conclusion: Conventional culture technique is the gold standard for diagnosis of pulmonary tuberculosis. Priority for tuberculosis control remains early detection and effective treatment of active cases of tuberculosis.
\end{abstract}

Key words: Tuberculosis, HIV, AFB Smear, Sputum, Non-Tuberculous Mycobacteria

\section{Introduction}

Tuberculosis is a major health problem of many developing countries including India [1]. About onethird of the total world's population is estimated to be infected by the causative agent Mycobacterium tuberculosis [2]. Tuberculosis manifests in individuals with suppressed immune system due to malnutrition, old age, diabetes mellitus and infections like HIV etc.

The incidence of tuberculosis has declined in developed countries with improved living conditions since the turn of twentieth century. With the emergence of HIV pandemic the steady decline in the incidence of

Manuscript received: $30^{\text {th }}$ March 2017

Reviewed: $8^{\text {th }}$ April 2017

Author Corrected: $17^{\text {th }}$ April 2017

Accepted for Publication: $24^{\text {th }}$ April 2017 tuberculosis was halted in developed Countries and in fact it is reversed and also there is an alarming increase in tuberculosis cases in developing and resource - poor countries [3].

Both tuberculosis and HIV infection have several features in common. Both of them cause severe morbidity and mortality. They afflict usually less previliged people. While tuberculosis is the leading cause killer of the world, HIV continues to infect millions of people around the globe [4].

HIV infection is considered to be the strongest risk factor for development of tuberculosis infection into active disease and tuberculosis is the commonest 
Research Article

opportunistic infection in persons infected with HIV. One out of three HIV infected persons die of tuberculosis.

Tuberculosis and Diabetes mellitus are common disorders and not surprisingly they co-exist. Recent studies noted that the risk of developing tuberculosis was higher in diabetics than in normal population [1].

With this background, this study was taken up to evaluate the prevalence of Tuberculosis among different risk group population like HIV, Diabetics and immunocompetent persons in and around Kakinada.

\section{Materials and Methods}

Study design: This was a prospective study carried out to determine the prevalence of TB among different risk groups like HIV, Diabetics and immunocompetent individuals. We collected sputum samples from 100 consecutive clinically suspected pulmonary tuberculosis patients. Modified petroffs method was used to decontaminate the sputum samples.

Microscopy was performed by Ziehl Neelson technique and culture was done using standard LJ solid media. We determined the prevalence of tuberculosis among different risk groups and attempted to study the isolation rates in smear negative cases. Culture on solid $\mathrm{LJ}$ medium was found to be more sensitive compared to sputum microscopy for the detection of Mycobacterium tuberculosis.

Setting: The present study was carried out by the department of Microbiology on patients attending Chest and TB diseases OP and patients admitted to Chest and TB diseases wards, Government General Hospital, Kakinada.

\section{Inclusion criteria}

1. Group IV category C of HIV patients attending Chest and TB OPD, with symptoms of pulmonary tuberculosis.

2. Diabetics admitted to chest and TB wards and patients attending Chest and TB diseases OPD, with symptoms of pulmonary tuberculosis.

3. Clinically suspected pulmonary tuberculosis cases attending chest and TB OPD who are otherwise normal and apparently immunocompetent.

\section{Exclusion criteria}

1. HIV positive cases who are recently diagnosed and not having any previous history of TB.

2. Patients who have discontinued treatment after diagnosis of pulmonary tuberculosis.

3. Patients with pulmonary tuberculosis who are already under treatment.

Participants : Specimen were collected from clinically suspected cases of tuberculosis in and around Kakinada from different risk groups like HIV /AIDS (50), Diabetics (25) and immunocompetent individuals (25).

Variables: Qualitative.

Data source: Clinical and demographic data were collected from patients attending chest and TB OPD and wards, Government general Hospital, Kakinada.

Bias: None.

Study size: 100 subjects.

Quantitative variables: None.

\section{Laboratory Methods:}

\section{Specimen Collection}

- Two sputum specimens were collected from the patient in sterile wide mouthed universal containers $(28 \mathrm{ml})$.

- One was an early morning collection of sputum and the other spot specimen.

- A volume of 5-10 ml was considered adequate.

Specimen Preparation- All the specimens were examined microscopically by using Ziehl- Neelsen's staining as per the standard protocol [5].The modified Petroff's technique was used for the decontamination of the sputum samples [6].

Mycobacterium culture- Commercially prepared LJ slants were used for the study (HiMedia). Two loops full of the decontaminated deposit was inoculated on the entire surface of 2 plain LJ slopes and one LJ slope containing PNB in a pre-sterilized inoculation hood, taking the necessary aseptic precautions. One plain LJ slant was covered, the other slant was incubated as such.The date of the inoculation was noted. The slopes were incubated at $37^{\circ} \mathrm{C}$, horizontally for the first $24 \mathrm{hrs}$ followed by vertical incubation. Check for growth was done every $24 \mathrm{hrs}$ during the first week followed by every week thereafter for 8 weeks. 
Reading of Cultures- Typical colonies of $\mathrm{M}$. tuberculosis are rough, crumbly, waxy, non-pigmented (cream coloured) and slow growers, i.e. only appearing two to three weeks after inoculation.

\section{Identification of Mycobacteria Tb Complex}

The positive cultures obtained after 8 weeks were identified as M. tuberculosis by -

a) Rate of growth: Growth appearing in less than one week was considered as rapid growth.MTB takes 34 weeks to grow.

b) Colour of the colony: MTB is buff coloured.Some atypical Mycobacteria (NTM ) produce pigment (Yellow colour ).

c) Colony appearance: MTB shows rough colony appearance. d) Growth test on PNB (Para-Nitro Benzoate): Growth is inhibited on Lowenstein Jensen medium containing PNB (500 ug/ml).

e) Nitrate reductase test: Mycobacterial tuberculosis reduces nitrate while M.bovis and BCG do not. M.tuberculosis is one of the strongest reducers of nitrate among the mycobacteria and helps in differentiating M. tuberculosis from the other Mycobacteria.

Statistical methods: Using SPSS software, Fishers exact test was applied to show whether there is association between smear microscopy and culture. Pvalue of $0.000,0.003$ and 0.0008 respectively were obtained for the the 3 risk groups namely HIV, Diabetics and Immunocompetent cases taken individually [Table 4]. These values of $\mathrm{p}$ are extremely statistically significant indicating that there is association between smear microscopy and culture.

\section{Results}

A total of 100 cases with clinical symptoms of pulmonary tuberculosis were studied of which 50 were HIV - positive individuals, 25 were diabetics and 25 were immunocompetent persons.

Among the study groups, maximum number of cases (66) were in the age-group of 20-40 yrs followed by $>40$ yrs. Male preponderance was observed in all age groups (Table 1). Male: Female ratio among patients from all the risk groups coinfected with TB is $3.5: 1$.

Table-1: Age and sex distribution of TB cases among different risk groups.

\begin{tabular}{|c|c|c|c|c|c|}
\hline Study group & Age & Males & Females & $\begin{array}{l}\text { No. of males } \\
\text { and females. }\end{array}$ & $\begin{array}{c}\text { Total \% of males } \\
\text { and females. }\end{array}$ \\
\hline \multirow[t]{3}{*}{ HIV-TB } & $<20$ & 2 & 0 & 2 & 4.88 \\
\hline & $20-40$ & 10 & 2 & 12 & 29.27 \\
\hline & $>\quad 40$ & 6 & 2 & 8 & 19.5 \\
\hline \multirow[t]{3}{*}{ Diabetes -TB } & $<20$ & 0 & 0 & 0 & 0 \\
\hline & $20-40$ & 2 & 0 & 2 & 4.88 \\
\hline & 40 & 0 & 0 & 0 & 0 \\
\hline \multirow[t]{3}{*}{ Immunocompetent -TB } & $<20$ & 2 & 1 & 3 & 7.31 \\
\hline & $20-40$ & 6 & 2 & 8 & 19.51 \\
\hline & $>\quad 40$ & 4 & 2 & 6 & 14.63 \\
\hline
\end{tabular}

Table-2: Sputum analysis among patients in different risk groups.

\begin{tabular}{|c|c|c|}
\hline $\begin{array}{c}\text { Study } \\
\text { Group }\end{array}$ & $\begin{array}{l}\text { Sputum } \\
\text { Positive }\end{array}$ & $\begin{array}{c}\text { Sputum } \\
\text { negative }\end{array}$ \\
\hline HIV positive(n=50) & $17(34 \%)$ & $33(66 \%)$ \\
\hline Diabetics $(\mathrm{n}=25)$ & $2(8 \%)$ & $23(92 \%)$ \\
\hline Immunocompetent $(\mathrm{n}=25)$ & $15(60 \%)$ & $10(40 \%)$ \\
\hline Total & $\mathbf{3 4}$ & $\mathbf{6 6}$ \\
\hline
\end{tabular}


Research Article

Table-3: Grading of AFB smears among patients in different risk groups.

\begin{tabular}{|c|c|c|c|}
\hline $\begin{array}{c}\text { AFB } \\
\text { Smear. }\end{array}$ & $\begin{array}{c}\text { HIV-positive } \\
\text { Cases. }\end{array}$ & Diabetics. & $\begin{array}{c}\text { Immunocompetent } \\
\text { persons. }\end{array}$ \\
\hline Negative & $33(66 \%)$ & $23(92 \%)$ & $10(40 \%)$ \\
\hline Scanty & $2(4 \%)$ & $0(0 \%)$ & $1(4 \%)$ \\
\hline $1+$ & $4(8 \%)$ & $1(4 \%)$ & $1(4 \%)$ \\
\hline $2+$ & $9(18 \%)$ & $1(4 \%)$ & $3(12 \%)$ \\
\hline $3+$ & $2(4 \%)$ & $0(0 \%)$ & $10(40 \%)$ \\
\hline Total & $\mathbf{5 0 ( 5 0 \% )}$ & $\mathbf{2 5 ( \% )}$ & $\mathbf{2 5 ( 2 5 \% )}$ \\
\hline
\end{tabular}

Table-4: Distribution of smear and culture isolates among different risk groups.

\begin{tabular}{|c|c|c|c|c|}
\hline & Study groups & $\begin{array}{c}\text { Culture } \\
\text { Positive }\end{array}$ & $\begin{array}{c}\text { Culture } \\
\text { Negative }\end{array}$ & $\begin{array}{c}\text { Significant } \\
\text { p-value }\end{array}$ \\
\hline $\mathbf{1 .}$ & HIV(n=50) & & & 0.0001 \\
\hline & Smear positive & 17 & 0 & \\
\hline $\mathbf{2 .}$ & Smear negative & 5 & 28 & \\
\hline & Diabetics(n=25) & 2 & 0 & 0.0033 \\
\hline & Smear positive & 0 & 23 & \\
\hline $\mathbf{3 .}$ & Smear negative & & & 0.0008 \\
\hline & Immunocompetent (n=25) & 15 & 0 & \\
\hline & Smear positive & 2 & 8 & \\
\hline
\end{tabular}

Out of a total of $50 \mathrm{HIV}$ positive cases, $22(44 \%)$ were found to be co-infected with tuberculosis. Among the 25 diabetic cases, $2(8 \%)$ were found to be co-infected with tuberculosis. Among symptomatic immunocompetent persons, out of 25 cases, $17(68 \%)$ were found to be suffering from pulmonary tuberculosis. Table 2 shows Sputum analysis among patients in different risk groups. Out of total 100 cases of symptomatic pulmonary tuberculosis, 34 (34\%) are sputum positive and $66(66 \%)$ are sputum negative. Maximum no. of sputum positive cases are among symptomatic immunocompetent persons $(60 \%)$ followed in order by HIV positive cases (34\%) and Diabetics $(8 \%)$.

Table 3 shows grading of AFB smears among patients in different risk groups. Among HIV positive patients , maximum number of smears were of grade $2+(18 \%)$ followed by $1+(8 \%)$ smears. Among diabetics with TB, smear positivity was $8 \%$ of which $4 \%$ smears were of grade $1+$ and $4 \%$ were grade 2 . Among immunocompetent with TB, maximum number of smears were of grade $3+$ followed by $2+(12 \%), 1+(4 \%)$ and scanty in that order.

Table 4 shows distribution of smear and culture isolates among different risk groups with symptoms of Pulmonary TB. Out of 22 isolates from HIV cases, 17(34\%) were both smear and culture positive and $5(10 \%)$ were smear negative and culture positive. Among diabetics, 2 (8\%) isolates were obtained which were both smear and culture positive.

Out of a total of 17 isolates from symptomatic immunocompetent patients, $15(60 \%)$ were both smear and culture positive and $2(8 \%)$ were smear negative and culture positive.

Table 4 shows distribution of smear negative, culture positive cases among different risk groups. Out of total 41 culture isolates, 7 isolates (18\%) were smear negative but culture positive. Among HIV cases co-infected with TB (22 isolates), 5 isolates $(10 \%)$ were smear negative but culture positive.

All the strains isolated were identified as Mycobacterium tuberculosis and no non-tuberculous mycobacteria spp (NTM) were isolated. Table 2 shows prevalence of NTM s and Mycobacterium tuberculosis among TB cases. 


\section{Discussion}

HIV pandemic has a major impact on tuberculosis incidence and mortality. Most of the developing countries have witnessed a dramatic rise in tuberculosis related deaths and hospitalization [7]. Incidence of HIV/TB co-infection in India is still progressing, though globally there is an overall decline. Firstly, India being an endemic country for TB, cases of reactivation is very high and secondly improper diagnosis and lack of treatment, especially among low socio-economic and underpreviliged population augments the problem [8]. A steady increase in TB prevalence among HIV patients in India has been reported by several authors $[9,10]$.

Patients with diabetes mellitus are also at a higher risk of tuberculosis. More recent studies noted that the risk of developing tuberculosis was 3-11 times greater in diabetics than in normal population. Neverthless there is a physiologic basis for the increased incidence of pulmonary tuberculosis in diabetics. Pulmonary tuberculosis was found to be more common in uncontrolled diabetics than in controlled diabetics. In the present study, tuberculosis co-infection among diabetics was found to be $8 \%$ which is in line with observations made in previous studies [11-15].

According to WHO death rate due to TB in India is very high among all other communicable diseases. So, an attempt was made in this study to estimate the prevalence of TB among symptomatic immunocompetent persons as well which was found to be $64 \%$. This was similar to the prevalence rate in other studies.

In the present study, maximum number of patients with TB co-infection among different risk groups are from the age group of 21-40 years with male preponderance. The occurrence of the disease in the age group of 21-40 years corresponds to the most productive age of an individual. Male preponderance could be due to more exposure in the community.

Similar results have been reported by S. Aktogu et al and Erdogmunsz in separate studies conducted on people living in large urban centres in the region of turkey [16]. As the main route of HIV transmission is through unprotected sex with an infected person, this is bound to occur in sexually active age group. Thus the incidence of TB among HIV positive patients is maximum in this age group and the outcome of the present study is also the same.
Similar incidence is reported by Theur et al [17] from California and Prasad et al [8] from India. These authors observed $52 \%$ and $48 \%$ of the HIV /TB coinfected patients in the age group of 21-40 years with male preponderance.

Smear positivity in sputum specimens among HIV patients, diabetics and immunocompetent persons was found to be $34 \%, 8 \%$ and $60 \%$ respectively.

In HIV-TB, sputum positivity is less common compared to HIV negative individuals, Several explanations are put forward for the same. Because of debility of HIVTB patients the sputum is not expectorated properly. Immunological impairement may lead to suppression of cough and there is no expectoration [18]. Cavitary disease was found to be significantly less frequent among the HIV infected patients which accounted for greater smear negativity among HIV-positive patients compared to seronegative patients [19]. The results are in line with observations made by previous authors.

Though sputum AFB microscopy is simple and cheap, sustaining its quality requires intense human support. Isolation of Mycobacterium tuberculosis from clinical samples is the confirmatory test for the diagnosis of tuberculosis. Hence, isolation of Mycobacterium tuberculosis by conventional culture technique has been attempted in this study.

Overall culture positivity from the sputum specimens was observed to be $41 \%$. Culture positivity rate of $30 \%$ has been documented by Shailaja et al [20].

The variation in culture- positivity of the specimen depends on several factors like the load of organisms in the specimen, technique of digestion of specimen, duration of treatment and antibiotics used.

In the present study, culture positivity rate in HIVpositive patients, Diabetics and immunocompetent persons was found to be $44 \%, 8 \%$ and $68 \%$ respectively. The present study also aimed to detect smear negative but culture positive patients which revealed a smear AFB negativity and culture positivity rates of $10 \%$ and $8 \%$ among HIV positive and immunocompetent persons respectively. The reason for this difference could be the fact that microscopic examination requires the presence of 10,000 to $1,00,000$ 
$\mathrm{org} / \mathrm{ml}$ for visualization of acid-fast bacilli whereas for culture even 10-100 bacilli are sufficient. In the present study, all the 41 culture positive cases, were identified as Mycobacterium tuberculosis and non-tuberculous mycobacteria sp.(NTM) were not isolated.

The variation in the incidence of NTM may be due to marked geographical variability both as regards to the prevalence of disease and also prevalence of Mycobacteria sp. in the community. Although majority of NTM are considered to be saprophytes, they have gained the status of potential pathogens among HIV positive patients due to their immunosuppressed status.

The isolation of NTM from a pulmonary source presents a diagnostic challenge as some NTM species that are generally considered non-pathogenic have been associated with pulmonary disease in the HIV infected host. [19].

The American Thoracic society (ATS) has published diagnostic criteria recommending repeated culture of specimens from non-sterile sites such as sputum before committing a patient to long term therapy with antibiotics. These guidelines recommend: 1) three positive sputum cultures with negative AFB smear results. 2) At least two positive sputum cultures and one positive AFB smear in the presence of compatible clinical and radiological features.

\section{Conclusion}

Conventional culture technique is the gold standard for diagnosis of pulmonary tuberculosis in comparision with smear detection using ziehl-Neelsen staining technique. All culture positive strains from sputum specimens were identified as Mycobacterium tuberculosis. No Non-tuberculous Mycobacteria species (NTM) were isolated. Priority for tuberculosis control remains early detection and effective treatment of active cases of tuberculosis and HIV targeting both the epidemics. Similarly an epidemic of diabetes mellitus is sweeping the country. And there is a growing amount of evidence that TB and diabetes are fuelling one another.

Funding: Nil, Conflict of interest: None initiated, Permission from IRB: Yes

\section{References}

1. Satya Sri S, History of tuberculosis in text book of pulmonary and extrapulmonary tuberculosis. New Delhi: Interprint; 1998.
2. Spence DP, Hotchkiss J, Williams CS, Davies PD. Tuberculosis and poverty. BMJ. 1993 Sep 25; 307 (6907) : 759-61.

3. Centers for Disease Control (CDC). Update on acquired immune deficiency syndrome (AIDS)--United States. MMWR Morb Mortal Wkly Rep. 1982 Sep 24; 31 (37):507-8, 513-4.

4. Drobniewski FA, Pozniak AL, Uttley AH. Tuberculosis and AIDS. J Med Microbiol. 1995 Aug; 43 (2):85-91.

5. Farnia PVM, Mohammadi F, Ghadiri Fard F, Doraghi M, Tabatabaee SJ, Zia Zarifi A, et al.Smear and microscopic. Mycobacteriology Research Center. 2009. Available from:http://fa.mrc.ac.ir/.

6. Farnia PVM, Mohammadi F, Ghadiri Fard F, Doraghi M, Tabatabaee SJ, Zia Zarifi A, et al.Culture Examination. Mycobacteriology Research Center.2009. Available from:www.mrc.ac.ir.

7. Gao L, Zhou F, Li X, Jin Q. HIV/TB co-infection in mainland China: a meta-analysis. PLoS One. 2010 May 20;5(5):e10736. doi: 10.1371/journal.pone.0010736.

8. Pal Ramprasad Balikaran, Singh pintu, Datta suprama. Incidence of Multi- Drug Resistant (MDR) and Extensive/ Extremely Drug resistant (XDR) Tuberculosis among HIV- positive patients in Mumbai. J BIOsci Tech, 2010; 4:135-143.

9. S.K.Agarwal, Aman Makhija,N.P.Singh, Anupam prakash and U.K. Baveja. Tuberculosis in HIV/AIDS patients in a tertiary care hospital in Delhi. Ind.J.Tub. 2003; 50:163.

10. A.Mahajan ,V.R. Tandon, S.verma, JB Singh, M.Sharma. Prevalence of tuberculosis, hepatitis B, hepatitis $\mathrm{C}$ and syphilis co-infections among HIV/AIDS patients. Indian J Med Microbiol 2008;26:196-7.

11. Kant S, Lata H, Natu SM, Mishra AK, Verma NS. Diabetes mellitus with pulmonary tuberculosis--a double trouble. J Indian Med Assoc. 2013 Mar;111 (3): 187-91.

12. Patel JC. Complications in 8793 cases of diabetes mellitus 14 years study in Bombay Hospital, Bombay, India. Indian J Med Sci. 1989 Jul;43(7):177-83. 
13. Alisjahbana B, van Crevel R, Sahiratmadja E, den Heijer M, Maya A, Istriana E, Danusantoso $H$, Ottenhoff TH, Nelwan RH, van der Meer JW. Diabetes mellitus is strongly associated with tuberculosis in Indonesia. Int J Tuberc Lung Dis. 2006 Jun; 10 (6): 696-700.

14. Ezung T, Devi NT, Singh NT, Singh TB. Pulmonary tuberculosis and diabetes mellitus--a study. J Indian Med Assoc. 2002 Jun;100(6):376, 378-9.

15. Mboussa J, Monabeka H, Kombo M, Yokolo D, Yoka-Mbio A, Yala F. [Course of pulmonary tuberculosis in diabetics]. Rev Pneumol Clin. 2003 Feb; 59 (1):39-44.

16. Aktoğu S, Yorgancioglu A, Cirak K, Köse T, Dereli SM. Clinical spectrum of pulmonary and pleural tuberculosis: a report of 5,480 cases. Eur Respir J. 1996 Oct; 9(10):2031-5.
17. Theuer CP, Hopewell PC, Elias D, Schecter GF, Rutherford GW, Chaisson RE. Human immunodeficiency virus infection in tuberculosis patients. J Infect Dis. 1990 Jul;162(1):8-12.

18. Klein NC, Duncanson FP, Lenox TH 3rd, Pitta A, Cohen SC, Wormser GP. Use of mycobacterial smears in the diagnosis of pulmonary tuberculosis in AIDS/ ARC patients. Chest. 1989 Jun;95(6):1190-2.

19. Sonia Khatter, Urvashi B.Singh, J .Arora, T.Rana and P.Seth. Mycobacterial infections in Human immunodeficiency virus seropositive patients : Role of non-tuberculous mycobacteria. Indian J Tuberc 2008; 55: 28-33.

20. Shailaja V.V, Pal A ,Mathur DR and Lakshmi V ; Prevalence of bacterial and fungal agents causing lower respiratory tract infections in patients with HIV infection, Ind J Med Microbiol,2004; 22,28-33.

\section{How to cite this article?}

K. Pragathi, R. Lakshmi Kumari, Durga Rani, Srinivas. Isolation of Mycobacterium tuberculosis from different risk groups in and around Kakinada. Trop J Path Micro 2017;3(2):142-148.doi: 10.17511/jopm.2017.i2.13. 